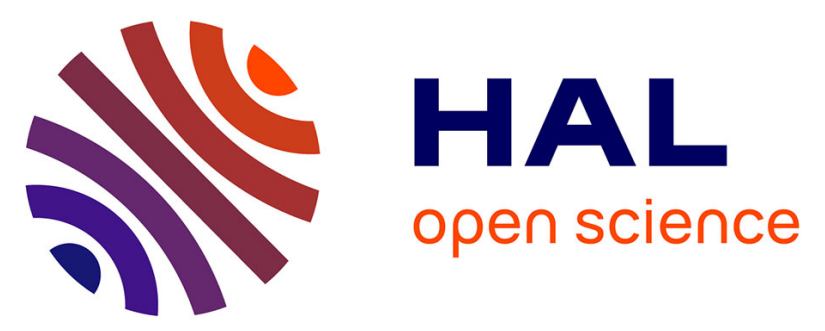

\title{
Stability and stabilizability of discrete-time dual switching systems with application to sampled-data systems
}

Lucien Etienne, Antoine Girard, Luca Greco

\section{- To cite this version:}

Lucien Etienne, Antoine Girard, Luca Greco. Stability and stabilizability of discrete-time dual switching systems with application to sampled-data systems. Automatica, 2019, 100, pp.388-395. 10.1016/j.automatica.2018.11.014 . hal-02286513

\section{HAL Id: hal-02286513 https://hal.science/hal-02286513}

Submitted on 13 Sep 2019

HAL is a multi-disciplinary open access archive for the deposit and dissemination of scientific research documents, whether they are published or not. The documents may come from teaching and research institutions in France or abroad, or from public or private research centers.
L'archive ouverte pluridisciplinaire HAL, est destinée au dépôt et à la diffusion de documents scientifiques de niveau recherche, publiés ou non, émanant des établissements d'enseignement et de recherche français ou étrangers, des laboratoires publics ou privés. 


\title{
Stability and stabilizability of discrete-time dual switching systems with application to sampled-data systems *
}

\author{
Lucien Etienne $^{\mathrm{a}}$, Antoine Girard ${ }^{\mathrm{b}}$, Luca Greco ${ }^{\mathrm{b}}$ \\ ${ }^{a}$ Ecole des Mines de Douai, 941 Rue Charles Bourseul, 59500 Douai, France \\ (e-mail: lucien.etienne@imt-lille-douai.fr). \\ ${ }^{\mathrm{b}}$ Laboratoire des Signaux et Systèmes (L2S), CNRS, CentraleSupélec, Université Paris-Sud, Université Paris-Saclay, \\ 3 rue Joliot-Curie, 91192 Gif-sur-Yvette, cedex, France \\ (e-mail: $\{$ antoine.girard, luca.greco\}@12s. centralesupelec.fr).
}

\begin{abstract}
In this paper, stability and stabilizability of discrete-time dual switching linear systems is investigated. The switched systems under consideration have two switching variables. One of them is stochastic, described by an underlying Markov chain; the other one can be regarded either as a deterministic disturbance or as a control input, leading to stability or stabilizability problems, respectively. For the considered class of systems, sufficient conditions for mean square stability (with or without control gain synthesis) and mean square stabilizability are provided in terms of matrix inequalities. When the stochastic switching is driven by an independent identically distributed sequence, we establish simpler conditions without additional conservatism. Then, it is shown how the proposed framework can be used to study aperiodic sampled-data systems with stochastic computation times. The results are illustrated on examples borrowed from the literature.
\end{abstract}

Key words: Switched systems, Markov jump linear systems, Matrix inequalities, Sampled-data systems, Scheduling.

\section{Introduction}

Switched systems constitute a powerful modeling framework making it possible to describe faithfully the interaction between physical systems and digital components such as computers or communication networks involved in modern control applications. Switched systems have been the subject of extensive research over the past two decades and is by now a well studied topic (see e.g. (Liberzon 2003, Lin \& Antsaklis 2009) and the references therein). The dynamics of a switched system is described by a collection of continuous dynamics, each of them describing the behavior of the system in some operating mode. The transition from one mode to the other is given by switching variables evolving according to some switching rule. The switching rule may be a priori unknown, in which case switching variables have to be regarded as disturbances (Ahmadi et al. 2014, Philippe et al. 2016). On the contrary, the switching rule may be regarded as control input (Geromel \& Colaneri 2006, Fi-

\footnotetext{
* This research was partially supported by Labex DigiCosme (project ANR-11-LABEX-0045-DIGICOSME) operated by ANR as part of the program "Investissement d'Avenir" Idex Paris Saclay (ANR-11-IDEX-0003-02).
}

acchini et al. 2016). When one assumes that the switching variables are driven by an underlying Markov chain, a linear switched system turns into a Markov jump linear system (Costa et al. 2006). In recent years, motivated by the study of networked control systems, new modeling frameworks have been proposed where only a subset of the switching variables are driven by a Markov chain, while for the others no statistical information is provided (Bolzern et al. 2010, 2013, 2016). Adopting the terminology introduced in these works, we refer to such systems as dual switching systems. Such models are useful in a wide range of application encompassing for instance supervisory control (Bolzern et al. 2013) or selftriggered control (as illustrated in the second part of this paper). In this paper, we deal with stability properties of discrete-time dual switching linear systems. The nonstochastic switching variables can be regarded either as deterministic disturbances or as control inputs, leading to stability or stabilizability problems, respectively.

In the first part of the paper, we present several sufficient conditions, in terms of matrix inequalities, for mean square stability (with or without control gain synthesis) and for mean square stabilizability. In particular, while Theorem 6 and Theorem 14 are similar to results 
presented in (Lutz \& Stilwell 2016) and (Bolzern et al. 2016), we make the following substantial contributions. We present linear matrix inequalities (LMIs) allowing us to synthesize stabilizing linear gains for discretetime dual switching linear systems. We also introduce two novel stabilizing switching rules, one stochastic and one deterministic, which provide possible alternatives to the min-switching strategy introduced in (Bolzern et al. 2016). In addition, for the special case when the stochastic switching sequence is driven by an independent identically distributed process, we establish much simpler stability and stabilizability conditions without additional conservatism. In the second part of the paper, we show how the considered framework can be used to study aperiodic sampled-data systems with stochastic computation times, under non-preemptive and preemptive scheduling policies. We propose a discrete-time dual switching linear system model for the system where stochastic switching variables describe varying computation times (due e.g. to varying loads on the computational platform) and other switching variables describe the prescription of the scheduler. We then show how stability of the system can be assessed using the theoretical results developed in the first part of the paper: when no assumption is made on the scheduling algorithm, besides lower and upper bounds for the time-varying sampling period (see e.g. (Al Khatib et al. 2017)), our stability conditions can be used; if one is also interested in designing the control algorithm, a self-triggered scheduling policy (see e.g. (Wang \& Lemmon 2009, Velasco et al. 2003)) can be synthesized using our stabilizability conditions.

The paper is organized as follows. Section 2 introduces the class of discrete-time dual switching linear systems and presents the stability and stabilizability problems under consideration. Section 3 presents several results for stability analysis of discrete-time dual switching linear systems, without or with control gain synthesis. Section 4 deals with stabilizability results and introduces several stabilizing switching rules. Section 5 shows the application of the framework to aperiodic sampled data systems. Finally, some concluding statements are provided in Section 6.

Notations: $\mathbb{R}^{+}$denotes the set of non-negative real numbers. $\mathbb{S}^{N}=\left\{\lambda \in \mathbb{R}^{N} \mid \forall i, \lambda_{i} \geq 0, \sum_{i=1}^{N} \lambda_{i}=1\right\}$ is the canonical unit simplex. For a vector $v,|v|$ denotes its Euclidean norm. For a vector or a matrix $v, v^{\prime}$ denotes its transpose. For a symmetric matrix $M, \lambda_{\min }(M)$ and $\lambda_{\max }(M)$ denote its smallest and largest eigenvalues, respectively. For two symmetric matrices $P$ and $Q$ we write $P>Q$ if $P-Q$ is positive definite $(P \geq Q$ if $P-Q$ is positive semidefinite). For integrable random variables $X, Y, \mathbb{E}[X]$ denotes the expectation of $X$ and $\mathbb{E}[X \mid Y]$ denotes the conditional expectation of $X$ knowing $Y$.

\section{Definitions and problem statement}

We consider discrete-time dual switching linear systems (Dt-DSLS), a class of switched linear systems with both stochastic and deterministic switching variables. Formally, let us consider the system:

$$
x_{k+1}=\bar{A}_{\left(d_{k}, s_{k}\right)} x_{k}+\bar{B}_{\left(d_{k}, s_{k}\right)} u_{k}, \quad k \in \mathbb{N},
$$

where the vectors $x_{k} \in \mathbb{R}^{n}, u_{k} \in \mathbb{R}^{p}$ and the switching variables $d_{k} \in \mathcal{D}=\left\{1, \ldots, N^{d}\right\}, s_{k} \in \mathcal{S}=\left\{1, \ldots, N^{s}\right\}$, for all $k \in \mathbb{N}$. Defining the real-valued control input as $u_{k}=K_{\left(d_{k}, s_{k}\right)} x_{k}$ and the matrices $A_{\left(d_{k}, s_{k}\right)}=\bar{A}_{\left(d_{k}, s_{k}\right)}+$ $\bar{B}_{\left(d_{k}, s_{k}\right)} K_{\left(d_{k}, s_{k}\right)}$ one has:

$$
x_{k+1}=A_{\left(d_{k}, s_{k}\right)} x_{k}, \quad k \in \mathbb{N} .
$$

We assume that the switching sequence $\left(s_{k}\right)_{k \in \mathbb{N}}$ is driven by a stochastic process defined on the probability space $(\Omega, \mathcal{F}, \mathrm{P})$ where $\Omega$ is the space of elementary events, $\mathcal{F}$ is the associated sigma-algebra and $P$ is the probability measure:

Definition 1 (Time homogeneous Markov chain) We say that $\left(s_{k}\right)_{k \in \mathbb{N}}$ is a time homogeneous Markov chain if for all $k \in \mathbb{N}$,

$$
\begin{array}{r}
\mathrm{P}\left(s_{k+1}=i_{k+1} \mid\right. \\
\left.s_{k}=i_{k}, \ldots, s_{0}=i_{0}\right) \\
=\mathrm{P}\left(s_{k+1}=i_{k+1} \mid s_{k}=i_{k}\right)=p_{i_{k} i_{k+1}} .
\end{array}
$$

We define the transition matrix $P=\left(p_{i j}\right)_{i, j \in \mathcal{S}}$. The initial probability distribution of $s_{k}$ is given by

$$
\pi_{0}:=\left(\mathrm{P}\left(s_{0}=1\right), \ldots, \mathrm{P}\left(s_{0}=N^{s}\right)\right) \in \mathbb{S}^{N^{s}} .
$$

Definition 2 (i.i.d. process) We say that $\left(s_{k}\right)_{k \in \mathbb{N}}$ is an independent and identically distributed (i.i.d.) process if for all $k \in \mathbb{N}$, for all $j \in \mathcal{S}, \mathrm{P}\left(s_{k}=j\right)=p_{j}$.

Note that i.i.d. processes form a special class of time homogeneous Markov chains where for all $i, j \in \mathcal{S}, p_{i j}=$ $p_{j}$. For i.i.d. processes, we will assume in the following (without loss of generality) that $p_{j}>0$ for all $j \in \mathcal{S}$. The switching sequence $\left(d_{k}\right)_{k \in \mathbb{N}}$ can be regarded either as a deterministic disturbance or as a control input. In the first case, assessing stability of system (2) is a robust stability problem:

Definition 3 (Mean square stability) $D t-D S L S$ (2) is mean square stable (MSS) if for any initial condition $x_{0} \in \mathbb{R}^{n}$, and any switching sequence $\left(d_{k}\right)_{k \in \mathbb{N}}$,

$$
\lim _{k \rightarrow \infty} \mathbb{E}\left[\left|x_{k}\right|^{2}\right]=0
$$

When $\left(d_{k}\right)_{k \in \mathbb{N}}$ is regarded as a control input, the problem at hand is a stabilization problem and requires the 
computation of a switching control law. We assume that at time step $k \geq 1$, the controller must choose the value of $d_{k}$ based on the knowledge of the current value of state $x_{k}$ and of the previous value of switching variables $d_{k-1}$, $s_{k-1}$. In this work, two kinds of switching control laws will be considered: deterministic and stochastic ones. In all generality, deterministic control laws can be seen as special cases of stochastic control laws. Deterministic control laws are given by $d_{0} \in \mathcal{D}$ and for all $k \geq 1$ :

$$
d_{k}=\theta\left(x_{k}, d_{k-1}, s_{k-1}\right),
$$

where $\theta: \mathbb{R}^{n} \times \mathcal{D} \times \mathcal{S} \rightarrow \mathcal{D}$. We also consider stochastic control laws where $\left(d_{k}\right)_{k \in \mathbb{N}}$ is a stochastic process with initial probability distribution $\pi_{d_{0}} \in \mathbb{S}^{N^{d}}$ and such that for all $k \geq 1, m \in \mathcal{D}, x \in \mathbb{R}^{n}, l \in \mathcal{D}, i \in \mathcal{S}$ :

$$
\mathrm{P}\left(d_{k}=m \mid x_{k}=x, d_{k-1}=l, s_{k-1}=i\right)=p_{d}(m, x, l, i) .
$$

Definition 4 (Mean square stabilizability) $D t$ -

$D S L S$ (2) is mean square stabilizable if there exists a switching control law, of the form (4) or (5), such that for any initial condition $x_{0} \in \mathbb{R}^{n}$, (3) holds.

Next, we recall a result that allows us to study stability properties using Lyapunov-like functions.

Theorem 5 ((Morozan 1968)) If for some $\alpha, \beta, \gamma>$ 0 and continuous functions $V_{k}: \mathbb{R}^{n} \rightarrow \mathbb{R}^{+}$, we have for all $k \in \mathbb{N}$,

$$
\begin{aligned}
& \text { H1: } \alpha|x|^{2} \leq V_{k}(x) \leq \beta|x|^{2}, \\
& \text { H2: } \mathbb{E}\left[V_{k+1}\left(x_{k+1}\right)\right] \leq \mathbb{E}\left[V_{k}\left(x_{k}\right)\right]-\gamma \mathbb{E}\left[\left|x_{k}\right|^{2}\right],
\end{aligned}
$$

then, (3) holds.

\section{Stability analysis of a Dt-DSLS}

\subsection{Sufficient stability conditions}

In this section, sufficient tractable conditions are provided to verify that a Dt-DSLS is MSS.

Theorem 6 If there exist symmetric matrices $M_{(l, i)} \in$ $\mathbb{R}^{n \times n}$ with $l \in \mathcal{D}, i \in \mathcal{S}$, such that the following LMIs are verified for all $l, m \in \mathcal{D}, i \in \mathcal{S}$ :

$$
\begin{aligned}
M_{(l, i)} & >0 \\
\sum_{j \in \mathcal{S}} p_{i j} A_{(l, i)}^{\prime} M_{(m, j)} A_{(l, i)}-M_{(l, i)} & <0
\end{aligned}
$$

then, Dt-DSLS (2), where $\left(s_{k}\right)_{k \in \mathbb{N}}$ is a time homogeneous Markov chain, is MSS.

By an appropriate reformulation of (2), the previous result can be seen as a special case of Theorem 4 in (Lutz
\& Stilwell 2016). Furthermore it can be seen as a special case of Theorem 2 in (Bolzern et al. 2016).

Remark 7 By considering $V_{k}\left(x_{k}\right)=x_{k}^{\prime} M_{\left(d_{k}, s_{k-1}\right)} x_{k}$ and using similar arguments, one can show that Dt-DSLS (2), where $\left(s_{k}\right)_{k \in \mathbb{N}}$ is a time homogeneous Markov chain, is MSS, if the following LMIs hold for all $l, m \in \mathcal{D}, i \in \mathcal{S}$ : $M_{(l, i)}>0$ and $\sum_{j \in \mathcal{S}} p_{i j} A_{(l, j)}^{\prime} M_{(m, j)} A_{(l, j)}-M_{(l, i)}<0$.

Remark 8 An alternative to study MSS of (2) is possible by adapting ideas of (Costa et al. 2006, Chapter 3). Consider the vector $\xi(k)=\left(\xi_{i}(k)\right)_{i \in \mathcal{S}}$ with $\xi_{i}(k)=$ $\operatorname{Vec}\left(\mathbb{E}\left[x_{k} x_{k}^{\prime} \chi_{\left\{s_{k}=i\right\}}\right]\right)$ where $V e c(M)$ is the vectorization of matrix $M$, and $\chi$ is the indicator function. One can show that $\xi$ is a trajectory of a deterministic switched linear systems of the form $\xi(k+1)=\mathcal{A}_{d_{k}} \xi(k)$. Since asymptotic stability of this system is equivalent to MSS of (2), the latter can be studied using existing approaches for switched linear systems (e.g. (Liberzon 2003, Chesi et al. 2012, Ahmadi et al. 2014)). The main drawback of this approach is that the dimension of the system to analyze is $n^{2} N^{s}$, which is significantly more than the dimension of (2).

\section{2 i.i.d. process}

An interesting special case of the previous study, is described when the elements of the stochastic sequence $\left(s_{k}\right)_{k \in \mathbb{N}}$ are independent and identically distributed. By imposing that for all $l \in \mathcal{D}, i \in \mathcal{S}, M_{(l, i)}=M_{l}$ and using the fact that for all $i, j \in \mathcal{S}, p_{i j}=p_{j}$, Remark 7 gives:

Corollary 9 If there exist symmetric matrices $M_{l} \in$ $\mathbb{R}^{n \times n}$ with $l \in \mathcal{D}$, such that the following LMIs are verified for all $l, m \in \mathcal{D}$ :

$\begin{aligned} M_{l} & >0, \\ \sum_{j \in \mathcal{S}} p_{j} A_{(l, j)}^{\prime} M_{m} A_{(l, j)}-M_{l} & <0,\end{aligned}$

then, Dt-DSLS (2), where $\left(s_{k}\right)_{k \in \mathbb{N}}$ is an i.i.d. process, is $M S S$.

Note that with respect to Theorem 6 and Remark 7 the number of variables and of inequalities is smaller. Moreover, this is without additional conservatism as shown by the following result:

Proposition 10 Let $p_{i j}=p_{j}$, for all $i, j \in \mathcal{S}$, if the LMIs (6) are feasible, then the LMIs (7) are feasible.

Proof. Let us assume that LMIs (6) are feasible for some symmetric matrices $M_{(l, i)}$ with $l \in \mathcal{D}, i \in \mathcal{S}$. Since by assumption $p_{i j}=p_{j}$ for all $i, j \in \mathcal{S},(6 \mathrm{~b})$ becomes for all $l, m \in \mathcal{D}, i \in \mathcal{S}$,

$$
\sum_{j \in \mathcal{S}} p_{j} A_{(l, i)}^{\prime} M_{(m, j)} A_{(l, i)}-M_{(l, i)}<0 .
$$


So, for all $l, m \in \mathcal{D}, i \in \mathcal{S}$,

$$
A_{(l, i)}^{\prime}\left(\sum_{j \in \mathcal{S}} p_{j} M_{(m, j)}\right) A_{(l, i)}-M_{(l, i)}<0
$$

Letting $Q_{m}=\left(\sum_{j \in \mathcal{S}} p_{j} M_{(m, j)}\right)>0$, one gets for all $l, m \in \mathcal{D}, i \in \mathcal{S}, A_{(l, i)}^{\prime} Q_{m} A_{(l, i)}-M_{(l, i)}<0$. So, for all $l, m \in \mathcal{D}$,

$$
\sum_{i \in \mathcal{S}} p_{i} A_{(l, i)}^{\prime} Q_{m} A_{(l, i)}-\sum_{i \in \mathcal{S}} p_{i} M_{(l, i)}<0 .
$$

Therefore, $\forall l, m \in \mathcal{D}, \sum_{i \in \mathcal{S}} p_{i} A_{(l, i)}^{\prime} Q_{m} A_{(l, i)}-Q_{l}<0$. Hence $Q_{l}$, with $l \in \mathcal{D}$ satisfy the LMIs (7).

Since the conditions of Theorem 6 imply the conditions of Corollary 9 and the numbers of matrix variables and of matrix inequalities in Corollary 9 are smaller, there is no benefit of checking the LMIs of Theorem 6 instead of those of Corollary 9 when $\left(s_{k}\right)_{k \in \mathbb{N}}$ is an i.i.d. process.

\subsection{Gain synthesis}

In the previous sections, we established sufficient conditions for mean square stability in terms of LMIs. In this section, from these results, we derive LMI conditions making it possible to synthesize the input gains $K_{(l, i)}$ for $l \in \mathcal{D}, i \in \mathcal{S}$ rendering Dt-DSLS (2) MSS. The results are proven in the Appendix.

Theorem 11 If there exists a set of symmetric matrices $Q_{(l, i)} \in \mathbb{R}^{n \times n}$ and of matrices $L_{(l, i)} \in \mathbb{R}^{p \times n}, S_{l} \in \mathbb{R}^{n \times n}$, $l \in \mathcal{D}, i \in \mathcal{S}$, such that the following set of LMIs is verified for all $l, m \in \mathcal{D}$ and $i \in \mathcal{S}$,

$Q_{(l, i)}>0$,

$$
\left[\begin{array}{c|c}
-S_{m}-S_{m}^{\prime}+\sum_{j \in \mathcal{S}} p_{i j} Q_{(m, j)} & \bar{A}_{(l, i)} S_{l}^{\prime}+\bar{B}_{(l, i)} L_{(l, i)} \\
\hline \star & -Q_{(l, i)}
\end{array}\right]<0,
$$

then, LMIs (6) hold with $M_{(l, i)}=S_{l}^{-1} Q_{(l, i)}\left(S_{l}^{\prime}\right)^{-1}$ and input gains $K_{(l, i)}=L_{(l, i)}\left(S_{l}^{\prime}\right)^{-1}$.

Note that in the previous results, the resulting control gains $K_{(l, i)}$ depend on both switching variables. Imposing new constraints on LMI variables $L_{(l, i)}$, and $S_{l}$ in (8) allows us to obtain control gains $K_{(l, i)}$ of the form $K_{l}, K_{i}$ or $K$. When the switching sequence $\left(s_{k}\right)_{k \in \mathbb{N}}$ is an i.i.d. stochastic process, one can use the following result:

Proposition 12 If there exists a set of symmetric matrices $Q_{l} \in \mathbb{R}^{n \times n}, W_{(l, i)} \in \mathbb{R}^{n \times n}$, and of matrices $L_{(l, i)} \in \mathbb{R}^{p \times n}, S_{l} \in \mathbb{R}^{n \times n}, l \in \mathcal{D}, i \in \mathcal{S}$ such that the following set of LMIs is verified for all $l, m \in \mathcal{D}$ and $i \in \mathcal{S}$,

$$
\begin{aligned}
& Q_{l}>0, \\
& {\left[\begin{array}{c|c}
-S_{m}-S_{m}^{\prime}+Q_{m} & \bar{A}_{(l, i)} S_{l}^{\prime}+\bar{B}_{(l, i)} L_{(l, i)} \\
\hline \star & -W_{(l, i)}
\end{array}\right]<0,} \\
& \sum_{j \in \mathcal{S}} p_{j} W_{(l, j)}-Q_{l} \leq 0,
\end{aligned}
$$

then, LMIs (7) hold with $M_{l}=S_{l}^{-1} Q_{l}\left(S_{l}^{\prime}\right)^{-1}$ and input gains $K_{(l, i)}=L_{(l, i)}\left(S_{l}^{\prime}\right)^{-1}$.

Then, the following result shows that, when the switching sequence $\left(s_{k}\right)_{k \in \mathbb{N}}$ is an i.i.d. process, conditions given by Proposition 12 are not more conservative than those given by Theorem 11:

Proposition 13 Let $p_{i j}=p_{j}$, for all $i, j \in \mathcal{S}$, if the LMIs (8) are feasible, then the LMIs (9) are feasible.

\subsection{Discussion}

Table 1 provides a comparison of the different LMIs in term of computational complexity. When the switching sequence $\left(s_{k}\right)_{k \in \mathbb{N}}$ is an i.i.d. process, a significant complexity reduction is enabled by the use of Corollary 9 , with no additional conservatism with respect to Theorem 6, as established by Proposition 10. For gain synthesis, the LMIs given by Proposition 12 are also slightly better in computational complexity than those of Theorem 11. However, one might prefer solving the former since they are less conservative than the latter, as established in Proposition 13.

\section{Stabilizability of Dt-DSLS}

Next, we consider the problem of stabilization of a DtDSLS by switching. The goal of stabilization is to find a control law for the switching sequence $\left(d_{k}\right)_{k \in \mathbb{N}}$ of the form (4) or (5), which renders (2) MSS.

\begin{tabular}{|l||c|c|c|c|}
\hline \multicolumn{1}{|c||}{} & \multicolumn{2}{c|}{ Stability analysis } & \multicolumn{2}{c|}{ Gain synthesis } \\
\hline$\left(s_{k}\right)_{k \in \mathbb{N}}$ & M.C. & i.i.d. & M.C. & i.i.d. \\
\hline Result & Th.6 & Cor.9 & Th.11 & Prop.12 \\
\hline \hline$n \times n$ M.V. & $N^{d} N^{s}$ & $N^{d}$ & $N^{d} N^{s}$ & $N^{d} N^{s}$ \\
$p \times n$ M.V. & 0 & 0 & $N^{d} N^{s}$ & $N^{d} N^{s}$ \\
\hline$n \times n$ M.I. & $\left(N^{d}\right)^{2} N^{s}$ & $\left(N^{d}\right)^{2}$ & $N^{d} N^{s}$ & $N^{d}$ \\
$2 n \times 2 n$ M.I. & 0 & 0 & $\left(N^{d}\right)^{2} N^{s}$ & $\left(N^{d}\right)^{2} N^{s}$ \\
\hline
\end{tabular}

Table 1

Complexity of LMIs established in Section 3: orders of the numbers of Matrix Variables (M.V.) and Matrix Inequalities (M.I.). 


\subsection{Sufficient stabilizability conditions}

In this section, sufficient stabilizability conditions are provided. Checking the stability conditions amounts to solving a set of Bilinear Matrix Inequalities (BMIs), which is in general a challenging task. Let us remind that a row stochastic matrix $\Pi$ in $\mathbb{R}^{N \times N}$ is a matrix where each row is an element of $\mathbb{S}^{N}$.

Theorem 14 If there exist symmetric matrices $M_{(l, i)} \in$ $\mathbb{R}^{n \times n}$, with $l \in \mathcal{D}, i \in \mathcal{S}$ and a row stochastic matrix $\Pi=\left(\pi_{l m}\right)_{l, m \in \mathcal{D}}$ such that the following BMIs are verified for all $l \in \mathcal{D}, i \in \mathcal{S}$ :

$$
\begin{aligned}
M_{(l, i)} & >0 \\
\sum_{j \in S} p_{i j} A_{(l, j)}^{\prime}\left(\sum_{m \in \mathcal{D}} \pi_{l m} M_{(m, j)}\right) A_{(l, j)}-M_{(l, i)} & <0
\end{aligned}
$$

then, Dt-DSLS (2), where $\left(s_{k}\right)_{k \in \mathbb{N}}$ is a time homogeneous Markov chain, is mean square stabilizable using the deterministic switching control law given by:

$$
d_{k}=\underset{l \in \mathcal{D}}{\arg \min }\left(x_{k}^{\prime} M_{\left(l, s_{k-1}\right)} x_{k}\right) \text {. }
$$

Proof. The proof is easily adapted from (Bolzern et al. 2016) with the slight difference that here $V_{k}\left(x_{k}\right)=$ $x_{k}^{\prime} M_{\left(d_{k}, s_{k-1}\right)} x_{k}$ instead of $V_{k}\left(x_{k}\right)=x_{k}^{\prime} M_{\left(d_{k}, s_{k}\right)} x_{k}$.

Let us remark that the different choice of Lyapunov function allows us to propose a switching control law of the form (4), while with the control law of (Bolzern et al. 2016), the knowledge of $s_{k}$ (instead of $s_{k-1}$ ) is needed to determine $d_{k}$. Avoiding this requirement will be useful when considering stabilization of sampled data systems.

By specializing the BMIs of Theorem 14, imposing that for all $l \in \mathcal{D}, i \in \mathcal{S}, M_{(l, i)}=M_{l}$, one obtains the following conditions when the switching sequence $\left(s_{k}\right)_{k \in \mathbb{N}}$ is an i.i.d. process:

Corollary 15 If there exist symmetric matrices $M_{l} \in$ $\mathbb{R}^{n \times n}$, with $l \in \mathcal{D}$ and a row stochastic matrix $\Pi=$ $\left(\pi_{l m}\right)_{l, m \in \mathcal{D}}$ such that the following BMIs are verified for all $l \in \mathcal{D}:$

$\begin{aligned} M_{l} & >0, \\ \sum_{j \in S} p_{j} A_{(l, j)}^{\prime}\left(\sum_{m \in \mathcal{D}} \pi_{l m} M_{m}\right) A_{(l, j)}-M_{l} & <0,\end{aligned}$

then, Dt-DSLS (2), where $\left(s_{k}\right)_{k \in \mathbb{N}}$ is an i.i.d. process, is mean square stabilizable. using the deterministic switching control law given by:

$$
d_{k}=\underset{l \in \mathcal{D}}{\arg \min }\left(x_{k}^{\prime} M_{l} x_{k}\right)
$$

One can check that the number of matrix variables and of matrix inequalities is much smaller in Corollary 15 than in Theorem 14: $N^{d}$ matrices $M_{l}$ instead of $N^{d} N^{s}$ matrices $M_{(l, i)}$, and $2 N^{d}$ instead of $N^{d} N^{s}$ matrix inequalities. Moreover, when the switching sequence $\left(s_{k}\right)_{k \in \mathbb{N}}$ is an i.i.d. process and under mild assumptions there is no loss of generality in solving (12) instead of (10), as shown by the following result, which is proven in the Appendix.

Proposition 16 Let $p_{i j}=p_{j}$, for all $i, j \in \mathcal{S}$, and let us assume that for all $l \in \mathcal{D}$, there exists $i \in \mathcal{S}$ such that $A_{(l, i)}$ is invertible. If the BMIs (10) are feasible, then the BMIs (12) are feasible.

\subsection{Alternative switching control laws}

In this section, we provide both stochastic and deterministic alternative switching control laws to (11) that guarantee mean square stability under the same assumptions as in Theorem 14. The first one is a stochastic control law of the form (5):

Proposition 17 Under the assumptions of Theorem 14, the Dt-DSLS (2), where $\left(s_{k}\right)_{k \in \mathbb{N}}$ is a time homogeneous Markov chain, is mean square stabilizable using the stochastic switching control law given by:

$$
\mathrm{P}\left(d_{k+1}=m \mid d_{k}=l\right)=\pi_{l m} .
$$

Proof. Considering $V_{k}\left(x_{k}\right)=x_{k}^{\prime} M_{\left(d_{k}, s_{k-1}\right)} x_{k}$, (10a) implies that condition H1 of Theorem 5 is verified. Let us denote $\Delta_{k}=V_{k+1}\left(x_{k+1}\right)-V_{k}\left(x_{k}\right)$, and $I_{k}=\left\{d_{k}=\right.$ $\left.l, s_{k-1}=i, x_{k}=x\right\}$. Then, along the trajectory of (2), with the switching control law given by (14), one has:

$$
\begin{aligned}
\mathbb{E} & {\left[\Delta_{k} \mid I_{k}\right]=\mathbb{E}\left[x^{\prime}\left(A_{\left(l, s_{k}\right)}^{\prime} M_{\left(d_{k+1}, s_{k}\right)} A_{\left(l, s_{k}\right)}-M_{(l, i)}\right) x \mid I_{k}\right] } \\
& =\sum_{m \in \mathcal{D}} \pi_{l m} \mathbb{E}\left[x^{\prime}\left(A_{\left(l, s_{k}\right)}^{\prime} M_{\left(m, s_{k}\right)} A_{\left(l, s_{k}\right)}-M_{(l, i)}\right) x \mid I_{k}\right] \\
& =\sum_{j \in \mathcal{S}} p_{i j} \sum_{m \in \mathcal{D}} \pi_{l m}\left(x^{\prime}\left(A_{(l, j)}^{\prime} M_{(m, j)} A_{(l, j)}-M_{(l, i)}\right) x\right) .
\end{aligned}
$$

Therefore, by BMI (10b), there exists $\gamma>0$ such that

$$
\mathbb{E}\left[\Delta_{k}+\gamma\left|x_{k}\right|^{2} \mid I_{k}\right]=E\left[\Delta_{k} \mid I_{k}\right]+\gamma|x|^{2} \leq 0
$$

which implies that $\mathbb{E}\left[\Delta_{k}+\gamma\left|x_{k}\right|^{2}\right] \leq 0$. Hence, condition H2 in Theorem 5 holds. It follows that the DtDSLS (2) using the stochastic switching control law (14) is MSS.

The second alternative control law is deterministic of the form (4). Under the assumptions of Theorem 14, there 
exists $\gamma>0$ such that for all $l \in \mathcal{D}$ and $i \in \mathcal{S}$

$$
\sum_{j \in S} p_{i j} A_{(l, j)}^{\prime}\left(\sum_{m \in \mathcal{D}} \pi_{l m} M_{(m, j)}\right) A_{(l, j)}-M_{(l, i)} \leq-\gamma I .
$$

Proposition 18 Under the assumptions of Theorem 14, the Dt-DSLS (2), where $\left(s_{k}\right)_{k \in \mathbb{N}}$ is a time homogeneous Markov chain, is mean square stabilizable using the deterministic switching control law given by:

$$
d_{k}=\max \left\{l \in \mathcal{D} \mid x_{k}^{\prime} Q_{\left(l, s_{k-1}\right)} x_{k} \leq \min _{r \in \mathcal{D}} x_{k}^{\prime} M_{\left(r, s_{k-1}\right)} x_{k}\right\}
$$

where for $l \in \mathcal{D}, i \in \mathcal{S}$,

$$
Q_{(l, i)}=\sum_{j \in \mathcal{S}} p_{i j} A_{(l, j)}^{\prime}\left(\sum_{m \in \mathcal{D}} \pi_{l m} M_{(m, j)}\right) A_{(l, j)}+\gamma I .
$$

Proof. We first show that the control law is welldefined. For $k \in \mathbb{N}$, let $r_{k}=\arg \min _{r \in \mathcal{D}}\left\{x_{k}^{\prime} M_{\left(r, s_{k-1}\right)} x_{k}\right\}$. By $(15), x_{k}^{\prime} Q_{\left(r_{k}, s_{k-1}\right)} x_{k} \leq \min _{r \in \mathcal{D}} x_{k}^{\prime} M_{\left(r, s_{k-1}\right)} x_{k}$. Hence, the set

$$
\left\{l \in \mathcal{D} \mid x_{k}^{\prime} Q_{\left(l, s_{k-1}\right)} x_{k} \leq \min _{r \in \mathcal{D}} x_{k}^{\prime} M_{\left(r, s_{k-1}\right)} x_{k}\right\} \neq \emptyset .
$$

and the control law (16) is well-defined. Then, let $V_{k}\left(x_{k}\right)=\min _{r \in \mathcal{D}}\left\{x_{k}^{\prime} M_{\left(r, s_{k-1}\right)} x_{k}\right\}$. Note that (10a) implies that H1 of Theorem 5 is verified. Moreover, by denoting $\Delta_{k}=V_{k+1}\left(x_{k+1}\right)-V_{k}\left(x_{k}\right)$, and $I_{k}=\left\{x_{k}=\right.$ $\left.x, s_{k-1}=i\right\}$, we have

$$
\begin{aligned}
& \mathbb{E}\left[V_{k+1}\left(x_{k+1}\right) \mid I_{k}\right]=\mathbb{E}\left[\min _{r \in \mathcal{D}}\left\{x_{k+1}^{\prime} M_{\left(r, s_{k}\right)} x_{k+1}\right\} \mid I_{k}\right] \\
& \leq \mathbb{E}\left[\sum_{r \in \mathcal{D}} \pi_{d_{k}, r}\left(x_{k+1}^{\prime} M_{\left(r, s_{k}\right)} x_{k+1}\right) \mid I_{k}\right] \\
& \leq \mathbb{E}\left[x^{\prime} A_{\left(d_{k}, s_{k}\right)}^{\prime}\left(\sum_{r \in \mathcal{D}} \pi_{d_{k}, r} M_{\left(r, s_{k}\right)}\right) A_{\left(d_{k}, s_{k}\right)} x \mid I_{k}\right] \\
& \leq \mathbb{E}\left[\sum_{j \in \mathcal{S}} p_{i j}\left(x^{\prime} A_{\left(d_{k}, j\right)}^{\prime}\left(\sum_{r \in \mathcal{D}} \pi_{d_{k}, r} M_{(r, j)}\right) A_{\left(d_{k}, j\right)} x\right) \mid I_{k}\right] \\
& \leq \mathbb{E}\left[x^{\prime} Q_{\left(d_{k}, i\right)} x-\gamma|x|^{2} \mid I_{k}\right] \\
& \leq \mathbb{E}\left[\min _{r \in \mathcal{D}} x^{\prime} M_{(r, i)} x-\gamma|x|^{2} \mid I_{k}\right]
\end{aligned}
$$

where the last inequality comes from (16). Then, since

$$
\mathbb{E}\left[\min _{r \in \mathcal{D}} x^{\prime} M_{(r, i)} x-\gamma|x|^{2} \mid I_{k}\right]=\mathbb{E}\left[V_{k}\left(x_{k}\right)-\gamma\left|x_{k}\right|^{2} \mid I_{k}\right]
$$

it follows that $\mathbb{E}\left[\Delta_{k}+\gamma\left|x_{k}\right|^{2} \mid I_{k}\right] \leq 0$, which implies that $\mathbb{E}\left[\Delta_{k}+\gamma\left|x_{k}\right|^{2}\right] \leq 0$. Hence, condition H2 in Theorem 5 holds. It follows that the Dt-DSLS (2) using the deterministic switching control law (16) is MSS.

\subsection{Discussion}

It is noticeable that all control laws, while they have very distinctive interpretation and do not rely on the same amount of information, stabilize Dt-DSLS (2) under the same assumptions, which is that there is a solution to the set of BMIs (10). The fact that identical BMI conditions can lead to different stability results, with radically different meaning, has been considered in (Geromel \& Colaneri 2006) in the context of discrete-time switched systems, while the analogy between Lyapunov Metzler stabilization and Lyapunov Metzler analysis is discussed in (Bolzern \& Colaneri 2015) in the context of positive switched systems. Depending on the information structure and the stabilizability objective it might be preferable to make a stronger distinction between different switching strategy, see for instance (Geromel et al. 2016, Bolzern \& Colaneri 2015).

The control law given by (11) tries to make the Lyapunov function decrease as fast as possible, while (16) applies the largest mode for which the Lyapunov function is expected to decrease. Control law (16) appears to be useful when the modes are ordered by some priority relation, which states that larger modes are preferred to smaller ones. An example of application of this strategy will be shown in the next section. Note that (11) and (16) require the knowledge of the current state $x_{k}$ and of the previous value of the stochastic switching variable $s_{k-1}$. Remarkably, when the switching sequence $\left(s_{k}\right)_{k \in \mathbb{N}}$ is an i.i.d. stochastic process, and the matrices $M_{(l, i)}=M_{l}, l \in \mathcal{D}, i \in \mathcal{S}$ (as in Corollary 15), the control laws (11) and (16) do not depend on the value of the stochastic switching variable anymore. We also proposed a stochastic stabilizing control law given by (14). The main advantage of this strategy is that its implementation does not require the knowledge of the state or of the stochastic switching variable. In practice, the convergence to the origin is slower using (14) than (11) and the average value of $d_{k}$ is smaller than with (16). However, in case of sensor failure it can be useful to switch from control laws (11) or (16) to (14) to keep providing stability guarantees.

\section{Application to sampled-data systems}

In this section, we show how the framework presented in the paper can be used in the context of sampled-data control systems.

\subsection{Modeling}

Let us consider the following sampled-data system:

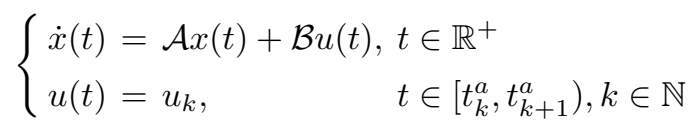


where $x(t) \in \mathbb{R}^{n}, u(t) \in \mathbb{R}^{p}$ and $\left(t_{k}^{a}\right)_{k \in \mathbb{N}}$ is the sequence of actuation instants. The sequence of input values $\left(u_{k}\right)_{k \in \mathbb{N}}$ will be specified later. Let $\left(t_{k}^{s}\right)_{k \in \mathbb{N}}$ be the sequence of sampling instants such that $t_{0}^{s}=0$ and $t_{k}^{s} \leq t_{k}^{a} \leq t_{k+1}^{s}$, and let $x_{k}=x\left(t_{k}^{s}\right)$ for $k \in \mathbb{N}$. Then, the sampled dynamics of (17) is given by:

$$
\begin{aligned}
x_{k+1}= & e^{\mathcal{A}\left(t_{k+1}^{s}-t_{k}^{s}\right)} x_{k}+\int_{t_{k}^{s}}^{t_{k+1}^{s}} e^{\mathcal{A}\left(t_{k+1}^{s}-\tau\right)} \mathcal{B} u(\tau) d \tau \\
= & e^{\mathcal{A}\left(t_{k+1}^{s}-t_{k}^{s}\right)} x_{k}+\int_{t_{k}^{s}}^{t_{k}^{a}} e^{\mathcal{A}\left(t_{k+1}^{s}-\tau\right)} d \tau \mathcal{B} u_{k-1} \\
& +\int_{t_{k}^{a}}^{t_{k+1}^{s}} e^{\mathcal{A}\left(t_{k+1}^{s}-\tau\right)} d \tau \mathcal{B} u_{k} \\
= & \Omega\left(\tau_{k}\right) x_{k}+\Omega\left(\tau_{k}-\delta_{k}\right) \Gamma\left(\delta_{k}\right) u_{k-1}+\Gamma\left(\tau_{k}-\delta_{k}\right) u_{k}
\end{aligned}
$$

where $\Omega(\rho)=e^{\mathcal{A} \rho}, \Gamma(\rho)=\int_{0}^{\rho} e^{\mathcal{A} \tau} d \tau \mathcal{B}$, and $\tau_{k}=t_{k+1}^{s}-$ $t_{k}^{s}, \delta_{k}=t_{k}^{a}-t_{k}^{s}$.

We assume that the sequence of input values is computed on a digital platform with limited resources and using atomic time slots of period $T$ :

- $s_{k} T$ is the time needed to compute the value of $u_{k}$ where $\left(s_{k}\right)_{k \in \mathbb{N}}$ is a time homogeneous Markov chain.

- $d_{k} T$ is the target inter-sampling times where $\left(d_{k}\right)_{k \in \mathbb{N}}$ is a sequence determined by a scheduler.

\subsubsection{Non-preemptive scheduling (NPS)}

In this scenario, we wait for computations to terminate (after $s_{k}$ time slots) before sampling again, even if this means that the next sampling instant is delayed (when $\left.d_{k}<s_{k}\right)$. In that case, the timing of events is given by

$$
\delta_{k}=s_{k} T, \tau_{k}=\max \left(d_{k}, s_{k}\right) T
$$

and the sequence of input values is given by $u_{k}=K_{1} x_{k}+$ $K_{2} u_{k-1}$, with by convention $u_{-1}=0$. Then, the sampled dynamics of (17) is described by the Dt-DSLS:

$$
X_{k+1}=A_{\left(d_{k}, s_{k}\right)} X_{k}
$$

where $X_{k}=\left[\begin{array}{c}x_{k} \\ u_{k-1}\end{array}\right], A_{\left(d_{k}, s_{k}\right)}=\bar{A}_{\left(d_{k}, s_{k}\right)}+\bar{B}_{\left(d_{k}, s_{k}\right)} K$ with $K=\left[\begin{array}{ll}K_{1} & K_{2}\end{array}\right]$ and

$\bar{A}_{\left(d_{k}, s_{k}\right)}=\left[\begin{array}{cc}\Omega\left(\tau_{k}\right) & \Omega\left(\tau_{k}-\delta_{k}\right) \Gamma\left(\delta_{k}\right) \\ 0 & 0\end{array}\right], \bar{B}_{\left(d_{k}, s_{k}\right)}=\left[\begin{array}{c}\Gamma\left(\tau_{k}-\delta_{k}\right) \\ I_{p}\end{array}\right]$.

\subsubsection{Preemptive scheduling (PS)}

In this scenario, if the computation did not finish by the next scheduled sampling instant (when $d_{k}<s_{k}$ ), the computation is interrupted, the input is kept at its previous value and sampling occurs at the targeted instant. In that case, the timing of events is given by

$$
\delta_{k}=\min \left(s_{k}, d_{k}\right) T, \tau_{k}=d_{k} T
$$

and the sequence of input values is given by

$$
u_{k}= \begin{cases}K_{1} x_{k}+K_{2} u_{k-1} & \text { if } s_{k} \leq d_{k} \\ u_{k-1} & \text { if } s_{k}>d_{k}\end{cases}
$$

with by convention $u_{-1}=0$. Then, the sampled dynamics of (17) is described by the Dt-DSLS:

$$
X_{k+1}=A_{\left(d_{k}, s_{k}\right)} X_{k}
$$

where $X_{k}=\left[\begin{array}{c}x_{k} \\ u_{k-1}\end{array}\right], A_{\left(d_{k}, s_{k}\right)}=\bar{A}_{\left(d_{k}, s_{k}\right)}+\bar{B}_{\left(d_{k}, s_{k}\right)} K$ with $K=\left[\begin{array}{ll}K_{1} & K_{2}\end{array}\right]$ and

$$
\begin{aligned}
& \bar{A}_{\left(d_{k}, s_{k}\right)}=\left\{\begin{array}{cl}
{\left[\begin{array}{cc}
\Omega\left(\tau_{k}\right) & \Omega\left(\tau_{k}-\delta_{k}\right) \Gamma\left(\delta_{k}\right)
\end{array}\right]} & \text { if } s_{k} \leq d_{k} \\
0 & 0 \\
{\left[\begin{array}{cc}
\Omega\left(\tau_{k}\right) \Gamma\left(\tau_{k}\right) \\
0 & I_{p}
\end{array}\right]} & \text { if } s_{k}>d_{k} .
\end{array}\right. \\
& \bar{B}_{\left(d_{k}, s_{k}\right)}=\left\{\begin{array}{cl}
{\left[\begin{array}{c}
\Gamma\left(\tau_{k}-\delta_{k}\right) \\
I_{p}
\end{array}\right]} & \text { if } s_{k} \leq d_{k} \\
{\left[\begin{array}{l}
0 \\
0
\end{array}\right]} & \text { if } s_{k}>d_{k}
\end{array}\right.
\end{aligned}
$$

In the following, we will use the results of the previous sections to analyze the dynamics of these systems.

\subsection{Exogenous scheduling policy}

We consider a first problem where the scheduler is an exogenous process, about which no statistical knowledge is available. Then, the scheduler is considered as a deterministic disturbance and stability analysis of Dt-DSLS (18) and (19) can be carried out using the results developed in Section 3.

As an illustration, we consider an example taken from (Tabuada 2007) given by $\mathcal{A}=\left[\begin{array}{cc}0 & 1 \\ -2 & 3\end{array}\right], \mathcal{B}=\left[\begin{array}{l}0 \\ 1\end{array}\right]$. We consider an atomic time slot $T=0.02$. The sequence of computation time $\left(s_{k}\right)_{k \in \mathbb{N}}$ is supposed to be an i.i.d. stochastic process with values in $\mathcal{S}=\{1, \ldots, 15\}$ and distribution shown in Figure 1. The target intersampling times $\left(d_{k}\right)_{k \in \mathbb{N}}$ are given by an exogenous scheduler with $d_{k} \in \mathcal{D}$ where $\mathcal{D}=\left\{d_{\min }, \ldots, d_{\text {max }}\right\}$ is a set of consecutive integers.

Using Proposition 12 one can synthesize stabilizing control gains $K$. The results are shown on Figures 2. One can conclude that non-preemptive schedulers are preferable from the point of view of stability. However, the timing behavior of preemptive schedules is much more predictable (the sequence of sampling instants $\left(t_{k}^{s}\right)_{k \in \mathbb{N}}$ 
is deterministic) and it is therefore much easier to deal with schedulability issues. In both cases, Figure 2 can be used to define specifications for designing scheduling protocols, which will guarantee stability of the sampled data system.

\subsection{Self-triggered scheduling policy}

We now assume that the scheduler is an endogenous process, which, based on available information, sets target inter-sampling times as large as possible (to use the computational resource parsimoniously) while providing stability guarantees. This control problem, can be tackled using the results developed in Section 4. More precisely, at sampling instant $t_{k}^{s}$, the information available to the scheduler consists in the value of the current sample $x_{k}$ with an estimation of the current load of the computational resource, given by the latest computation time $s_{k-1}$. The scheduler uses this information to set a target inter-sampling time $d_{k}$. This is an example of selftriggered scheduling (Wang \& Lemmon 2009), where the next sampling instant is scheduled dynamically based on information available at the current sample.

As an illustration, we consider the batch reactor taken from (Donkers et al. 2011) given by:

$\mathcal{A}=\left[\begin{array}{cccc}1.380 & -0.2077 & 6.715 & -5.676 \\ -0.5814 & -4.290 & 0 & 0.675 \\ 1.067 & 4.273 & -6.654 & 5.893 \\ 0.048 & 4.273 & 1.343 & -2.104\end{array}\right], \mathcal{B}=\left[\begin{array}{cc}0 & 0 \\ 5.7679 & 0 \\ 1.136 & -3.146 \\ 1.136 & 0\end{array}\right]$

with a time slot $T=0.02$. The control gains are

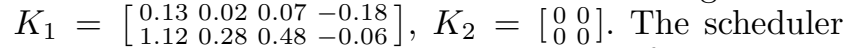
can choose target inter-sampling times from the set $\mathcal{D}=\{1, \ldots, 35\}$. The sequence of computation times $\left(s_{k}\right)_{k \in \mathbb{N}}$ is a time homogenous Markov chain, with $s_{0}=3$ and $s_{k} \in \mathcal{S}=\{3,10,17\}$, and transition matrix $P_{m}=$ $\left[\begin{array}{ccc}0.75 & 0.25 & 0 \\ 0.1 & 0.7 & 0.2 \\ 0 & 0.9 & 0.1\end{array}\right]$.

We first consider a non-preemptive scheduler. One can first notice that by choosing $d_{k}=10$ for all $k \in \mathbb{N}$, DtDSLS (18) is MSS. This means that BMIs (10) have solutions with the matrix $\Pi$ given by $\pi_{l m}=1$ if $m=10$ and $\pi_{l m}=0$ if $m \neq 10$. Note that once the matrix $\Pi$ is fixed (10) reduces to a set of LMIs, which are easily solved. We then use the control law given by (16), which tries to maximize the value of the next sampling instant while guaranteeing stability. Simulation results are shown on

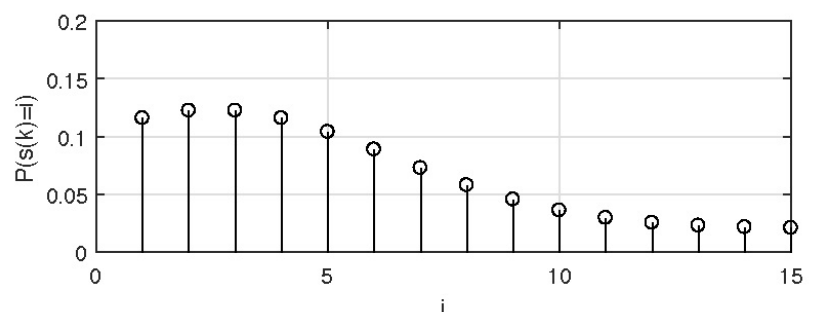

Fig. 1. Distribution of the i.i.d. stochastic process $\left(s_{k}\right)_{k \in \mathbb{N}}$.

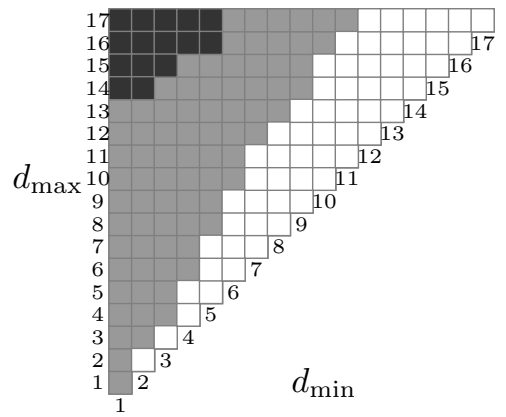

Fig. 2. Gain synthesis: each square represents a value of $d_{\min }$ and $d_{\text {max }}$; the square is white if LMIs (9) are feasible for (18) and (19) with $\mathcal{D}=\left\{d_{\min }, \ldots, d_{\max }\right\}$; gray if LMIs (9) are feasible for (18) but infeasible for (19) and black if LMIs (9) are infeasible for both (18) and (19).

Figure 5 (Left) for initial state $x(0)=[1,1,1,1]^{\prime}$ and $d_{0}=3$. We then consider a preemptive scheduler. With

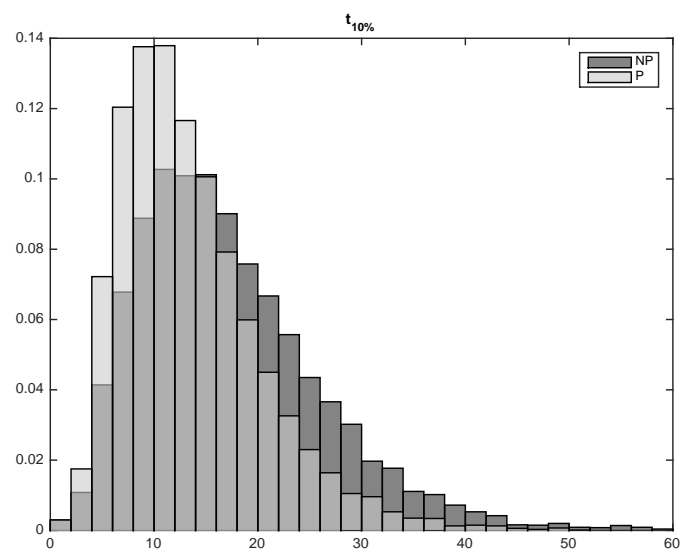

Fig. 3. Distribution of the $10 \%$ response time for self-triggered, non-preemptive (NP) scheduling and preemptive (P) scheduling. Results obtained on $10^{4}$ simulated trajectories with uniformly distributed initial conditions.

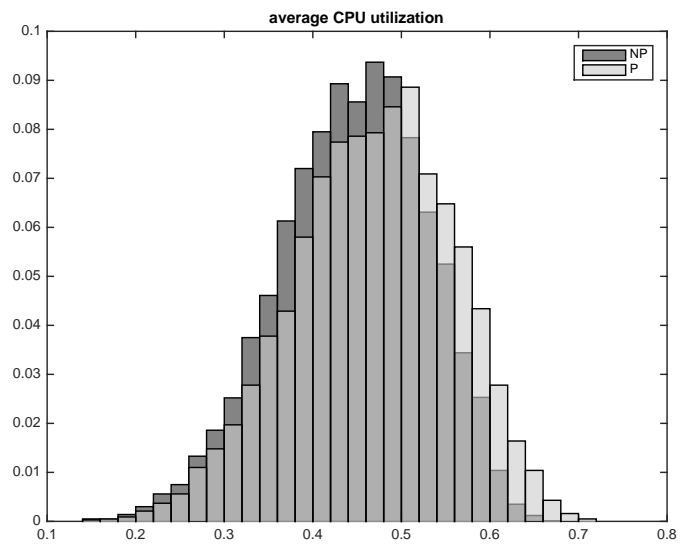

Fig. 4. Distribution of the CPU utilization for self-triggered, non-preemptive (NP) scheduling and preemptive (P) scheduling. Results obtained on $10^{4}$ thousand simulated trajectories with uniformly distributed initial conditions. 

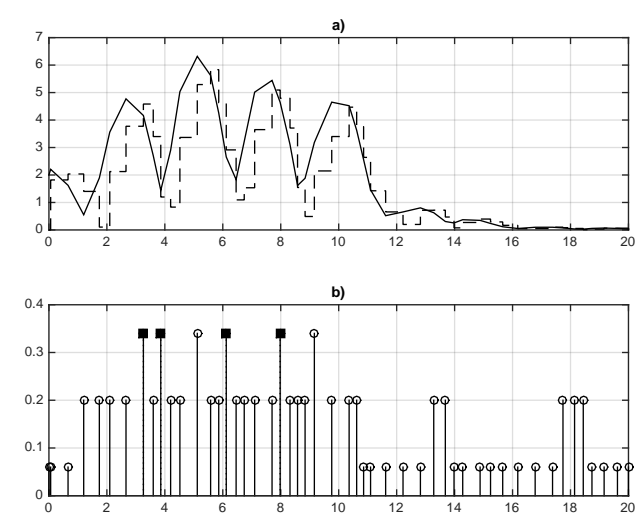
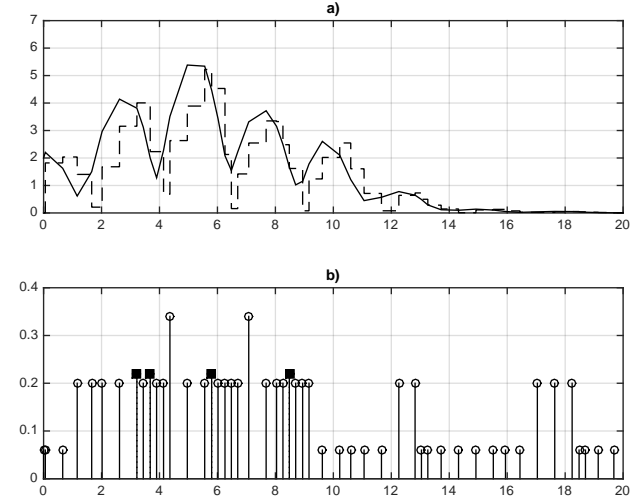

Fig. 5. Simulations for self-triggered scheduling, Left) NPS, Right PS: a) (plain) Norm of the state $|x(t)|$, (dashed) Norm of the input $|u(t)|$; b) Events: abscissa correspond to sampling instants $t_{k}^{s}$, ordinate correspond to computation times $\delta_{k}=t_{k}^{a}-t_{k}^{s}$; circles indicate that computation terminates before the next scheduled sampling, (NPS) squares indicate that the next sampling is delayed until computation terminates, (PS) squares indicate that the next sampling occurs before computation terminates.

the same constant strategy $d_{k}=10$, Dt-DSLS (19) is MSS. Then, BMIs (10) have solutions with the same matrix П. Simulation results using the control law given by (16) are shown on Figure 5 (Right) for initial state $x(0)=[1,1,1,1]^{\prime}$ and $d_{0}=3$. Note that for both NPS and PS in Figure 5, the average inter-sampling time is 0.4 while the stabilizing periodic time (corresponding to $\forall k \in \mathbb{N}, d_{k}=10$ ) is 0.2 . Thus using self-triggering scheduling in this case reduces sampling by $50 \%$.

We simulated $10^{4}$ trajectories with self-triggered scheduling for both NPS and PS with uniformly distributed initial states $x(0)$, and sequences $\left(s_{k}\right)$ generated by the Markov chain $P_{m}$. Figure 3 shows the distribution of the $10 \%$ response time, that is the time after which $|x(t)|$ remains smaller than $0.1 \times|x(0)|$. The $10 \%$ response time is a good indicator of the convergence speed. One can see that trajectories using PS generally converge faster than trajectories using NPS. In fact, the average response time is 13.5 in the former case and 17.2 in the latter case. Figure 4 shows the distribution of the CPU utilization, that is the portion of the time where the computational resource is used. PS use slightly more CPU than NPS. The average CPU utilization is 0.47 for the former and 0.44 for the latter. As a comparison, the periodic strategy $d_{k}=10$ gives a faster average response time of 4.9 (PS) and 5.1 (NPS) but the CPU is much more utilized 0.82 (PS) and 0.83 (NPS). We can see that self-triggered schedules succeed in achieving what they have been designed for, namely increasing the intersampling times and thus reducing the CPU utilization while preserving stability.

\section{Conclusion}

In this work, we have presented several sufficient conditions for stability and stabilizability of discrete-time dual switching linear systems. These conditions are computationally oriented and take the form of linear or bilinear matrix inequalities. Some of the results allow to design stabilizing control gains or stabilizing switching rules. Simpler conditions with no additional conservatism can be obtained when the stochastic switching variable is i.i.d. While in this paper only stability properties were considered, it should be possible to investigate, using similar Lyapunov based approaches, other classes of problems such has $H_{\infty}$ or $H_{2}$ analysis or synthesis (Bolzern et al. 2016). Finally, we have shown how the proposed framework allows us to model and analyze non-trivial scheduling problems in sampled-data systems, showing the relevance of dual switching linear systems for this type of applications.

\section{References}

Ahmadi, A. A., Jungers, R. M., Parrilo, P. A. \& Roozbehani, M. (2014), 'Joint spectral radius and pathcomplete graph Lyapunov functions', SIAM Journal on Control and Optimization 52(1), 687-717.

Al Khatib, M., Girard, A. \& Dang, T. (2017), 'Stability verification and timing contract synthesis for linear impulsive systems using reachability analysis', Nonlinear Analysis: Hybrid Systems 25, 211-226.

Bolzern, P. \& Colaneri, P. (2015), 'Positive Markov jump linear systems', Foundations and Trends in Systems and Control 2(3-4), 275-427.

URL: http://dx.doi.org/10.1561/2600000006

Bolzern, P., Colaneri, P. \& De Nicolao, G. (2010), 'Markov jump linear systems with switching transition rates: mean square stability with dwell-time', $A u$ tomatica 46(6), 1081-1088.

Bolzern, P., Colaneri, P. \& De Nicolao, G. (2013), 'Almost sure stability of Markov jump linear systems with deterministic switching', IEEE Transactions on Automatic Control 58(1), 209-214.

Bolzern, P., Colaneri, P. \& De Nicolao, G. (2016), 'Design of stabilizing strategies for discrete-time dual switching linear systems', Automatica 69, 93-100.

Chesi, G., Colaneri, P., Geromel, J. C., Middleton, R. \& Shorten, R. (2012), 'A nonconservative LMI condi- 
tion for stability of switched systems with guaranteed dwell time', IEEE Transactions on Automatic Control 57(5), 1297-1302.

Costa, O. L. V., Fragoso, M. D. \& Marques, R. P. (2006), Discrete-time Markov jump linear systems, Springer Science \& Business Media.

Donkers, M., Heemels, W., Van de Wouw, N. \& Hetel, L. (2011), 'Stability analysis of networked control systems using a switched linear systems approach', IEEE Transactions on Automatic control 56(9), 2101-2115.

Fiacchini, M., Girard, A. \& Jungers, M. (2016), 'On the stabilizability of discrete-time switched linear systems: Novel conditions and comparisons', IEEE Transactions on Automatic Control 61(5), 1181-1193.

Geromel, J. C. \& Colaneri, P. (2006), 'Stability and stabilization of discrete time switched systems', International Journal of Control 79(7), 719-728.

Geromel, J. C., Deaecto, G. S. \& Colaneri, P. (2016), 'Minimax control of Markov jump linear systems', International Journal of Adaptive Control and Signal Processing 30(8-10), 1152-1162.

Liberzon, D. (2003), Switching in Systems and Control, Springer Science \& Business Media.

Lin, H. \& Antsaklis, P. J. (2009), 'Stability and stabilizability of switched linear systems: a survey of recent results', IEEE Transactions on Automatic control 54(2), 308-322.

Lutz, C. C. \& Stilwell, D. J. (2016), 'Stability and disturbance attenuation for Markov jump linear systems with time-varying transition probabilities', IEEE Transactions on Automatic Control 61(5), 1413-1418.

Morozan, T. (1968), 'Stability of stochastic discrete systems', Journal of Mathematical Analysis and Applications 23(1), 1-9.

Philippe, M., Essick, R., Dullerud, G. E. \& Jungers, R. M. (2016), 'Stability of discrete-time switching systems with constrained switching sequences', Automatica 72, 242-250.

Tabuada, P. (2007), 'Event-triggered real-time scheduling of stabilizing control tasks', IEEE Transactions on Automatic Control 52(9), 1680-1685.

Velasco, M., Fuertes, J. \& Marti, P. (2003), The self triggered task model for real-time control systems, in '24th IEEE Real-Time Systems Symposium', Vol. 384.

Wang, X. \& Lemmon, M. D. (2009), 'Self-triggered feedback control systems with finite-gain $\mathcal{L}_{2}$ stability', IEEE Transactions on Automatic Control 54(3), 452467.

\section{Appendix}

\section{Sketch of Proof of Theorem 11}

Note that (8b) implies that for all $l \in \mathcal{D}, S_{l}$ is invertible. Thus, for all $l \in \mathcal{D}, i \in \mathcal{S}, M_{(l, i)}=S_{l}^{-1} Q_{(l, i)}\left(S_{l}^{\prime}\right)^{-1}>0$. Multiplying (8b) on the left by $\left[\begin{array}{cc}S_{m}^{-1} & 0 \\ 0 & S_{l}^{-1}\end{array}\right]$ and on the right by $\left[\begin{array}{cc}S_{m}^{-1} & 0 \\ 0 & S_{l}^{-1}\end{array}\right]^{\prime}$, using $K_{(l, i)}=L_{(l, i)}\left(S_{l}^{\prime}\right)^{-1}, M_{(l, i)}=$ $S_{l}^{-1} Q_{(l, i)}\left(S_{l}^{\prime}\right)^{-1}$ with $\bar{A}_{(l, i)}+\bar{B}_{(l, i)} K_{(l, i)}=A_{(l, i)}$, and denoting $G_{l}=S_{l}^{-1}$, we obtain for all $l, m \in \mathcal{D}, i \in \mathcal{S}$, $\left[\begin{array}{c|c}-G_{m}-G_{m}^{\prime}+\sum_{j \in \mathcal{S}} p_{i j} M_{(m, j)} & G_{m} A_{(l, i)} \\ \hline \star & -M_{(l, i)}\end{array}\right]<0$.

Multiplying on the left by $\left[A_{(l, i)}^{\prime}, I\right]$, and on the right by $\left[A_{(l, i)}^{\prime}, I\right]^{\prime}$, LMIs $(6)$ are obtained.

\section{Sketch of Proof of Proposition 12}

The proof is similar to the proof of Theorem 11.

\section{Sketch of Proof of Proposition 13}

Let us assume that the LMIs (8) are feasible. Let us denote $R_{m}=\sum_{j \in \mathcal{S}} p_{j} Q_{(m, j)}>0$. By definition of $R_{l}$ one has: $\sum_{j \in \mathcal{S}} p_{j} Q_{(l, j)}-R_{l}=0 \leq 0$. Then, renaming the variable appropriately $\left(Q_{(l, i)}\right.$ and $R_{l}$ becomes $W_{(l, i)}$ and $Q_{l}$ respectively) leads to LMIs (9).

\section{Sketch of Proof of Proposition 16}

Let us assume the BMIs (10) are feasible, for some $M_{(l, i)}>0, l \in \mathcal{D}, i \in \mathcal{S}$, and a row stochastic matrix $\Pi=\left(\pi_{l m}\right)_{l, m \in \mathcal{D}}$. Then, let us define for all $l \in \mathcal{D}, Q_{l}=\sum_{j \in \mathcal{S}} p_{j} A_{(l, j)}^{\prime}\left(\sum_{m \in \mathcal{D}} \pi_{l m} M_{(m, j)}\right) A_{(l, j)}$. Since there exists $j \in \mathcal{S}$ such that $A_{(l, j)}$ is invertible, then $Q_{l}>0$. Moreover (10b) gives for all $l \in$ $\mathcal{D}, i \in \mathcal{S}, Q_{l}-M_{(l, i)}<0$. Then, for all $r \in \mathcal{D}$, $i \in \mathcal{S}, \sum_{l \in \mathcal{D}} \pi_{r l}\left(Q_{l}-M_{(l, i)}\right)<0$. Since there exists $i \in \mathcal{S}$, such that $A_{(r, i)}$ is invertible, we have $\forall r \in$ $\mathcal{D}, \sum_{i \in \mathcal{S}} p_{i} A_{(r, i)}^{\prime}\left(\sum_{l \in \mathcal{D}} \pi_{r l}\left(Q_{l}-M_{(l, i)}\right)\right) A_{(r, i)}<0$. Let us remark that $\sum_{i \in \mathcal{S}} p_{i} A_{(r, i)}^{\prime}\left(\sum_{l \in \mathcal{D}} \pi_{r l}\left(Q_{l}-M_{(l, i)}\right)\right) A_{(r, i)}=$ $\sum_{i \in \mathcal{S}} p_{i} A_{(r, i)}^{\prime}\left(\sum_{l \in \mathcal{D}} \pi_{r l} Q_{l}\right) A_{(r, i)}-Q_{r}$. It follows that BMIs (12) are feasible for matrices $Q_{l}$, with $l \in \mathcal{D}$, and $\Pi$. 